\title{
Marked local and distant response of heavily treated breast cancer with cardiac metastases treated by combined low dose radiotherapy, low dose immunotherapy and hyperthermia: a case report
}

\author{
Mau-Shin Chi ${ }^{1}$, Jen-Hong Wu ${ }^{1}$, Suzun Shaw ${ }^{2}$, Ching-Jung Wu ${ }^{2}$, Liang-Kuang Chen ${ }^{3}$, Ho-Chi Hsu ${ }^{4}$, \\ Kwan-Hwa Chi ${ }^{1}$ \\ ${ }^{1}$ Department of Radiation Therapy and Oncology, Shin Kong Wu Ho-Su Memorial Hospital, Taipei, Taiwan; ${ }^{2}$ Department of Radiation Oncology, \\ Cathay General Hospital, Taipei, Taiwan; ${ }^{3}$ Department of Diagnostic Radiology, Shin-Kong Wu Ho-Su Memorial Hospital, Taipei, Taiwan; \\ ${ }^{4}$ Department of General Surgery, Shin Kong Wu Ho-Su Memorial Hospital, Taipei, Taiwan \\ Correspondence to: Ho-Chi Hsu. Department of General Surgery, Shin Kong Wu Ho-Su Memorial Hospital, Wenchang Rd., No. 95, Shihlin \\ District, Taipei, Taiwan. Email: M005284@ms.skh.org.tw; Kwan-Hwa Chi. Department of Radiation Therapy and Oncology, Shin Kong Wu Ho-Su \\ Memorial Hospital, Wenchang Rd., No. 95, Shihlin District, Taipei, Taiwan. Email: khchi45@gmail.com.
}

\begin{abstract}
Breast cancer (BC) with cardiac metastases (CMs) is often associated with poor prognosis due to late stage of diagnosis. Palliative radiotherapy (RT) for CMs is generally used for symptomatic treatment and to maintain normal cardiac function. Palliative RT with hyperthermia (HT) or immunotherapy have been reported to be effective in prolonging the overall survival and progression-free survival in metastatic patients. In this case report, we present a heavily pretreated 51-year-old lady of metastatic BC presented with recurrent right breast mass with progressive exertional dyspnea caused by symptomatic CM. She received combined palliative low-dose palliative RT [20 Gray (Gy) in 12 fractions], combined with lowdose chemotherapy, biweekly HT treatment course, and low-dose "double blockade" immunotherapy by ipilimumab $(0.3 \mathrm{mg} / \mathrm{kg})$ and nivolumab $(0.5 \mathrm{mg} / \mathrm{kg})$. The irradiated right chest tumors responded rapidly to treatment. Interestingly, unirradiated metastatic lesions outside the RT and HT treatment field also demonstrated a sustained abscopal response. She continued monthly low-dose immunotherapy in conjunction with HT after RT. The posttreatment cardiac echography disclosed considerably reduced pericardial effusions without cardiac wall motion abnormalities. She remained stable for more than 6 months with no notable treatment-related toxicities. The combination of low-dose RT, low-dose immunotherapy, and HT protocol appears to be a safe method with promising efficacy in metastatic BC patients.
\end{abstract}

Keywords: Cardiac metastases (CMs); radiotherapy (RT); immunotherapy; hyperthermia (HT); case report

Received: 14 July 2021; Accepted: 22 October 2021; Published: 30 December 2021.

doi: 10.21037/tro-21-16

View this article at: https://dx.doi.org/10.21037/tro-21-16

\section{Introduction}

Cardiac metastases (CMs) occur more frequently than is often expected. The incidence of CMs in metastatic cancers is up to $14.2 \%$, and they are most commonly derived from lung cancer (incidence of 36-39\%), followed by breast malignancies (incidence of $10-12 \%$ ) and hematologic malignancies (incidence of 10-21\%) (1). CMs are associated with a grave prognosis due to their generally late diagnosis at profound stages (2). CMs are clinically silent in general; nevertheless, they can be lethal when cardiac tamponade or a rapid accumulation of pericardiac effusion occurs (3). CM management strategies are generally aimed at symptomatic control and maintenance of normal cardiac function. The role of palliative radiotherapy (RT) in managing CMs is 
limited due to concerns over reported cardiac toxicities. The literature discussing RT as palliative treatment for CMs mostly involves case reports with varied radiation doses [6 to 54 Gray (Gy)] and response durations (4-6).

Breast cancer (BC) patients with CMs are often heavily pretreated before diagnosis, which means the remaining therapeutic window is narrow. Combinatory use of immunotherapy and chemotherapy was determined to be effective in prolonging overall survival and progression-free survival in patients with metastatic triple-negative BC (7). Hyperthermia (HT), known for its radio, or chemosensitization effect, combined with salvage RT has become an effective treatment method for recurrent BC (8). Modulated electrohyperthermia (mEHT) is a form of HT that transmits radiofrequency energy into the tumor cell membrane to produce an electric field. This field causes lymphocytic and dendritic cells to penetrate the tumor to achieve pronounced immunogenic cell death $(9,10)$. A combination of RT, mEHT, and immunotherapy may enable a rapid and sustained abscopal effect, as discussed in a retrospective study (11).

Herein, we report the case of a patient with metastatic BC with CMs who achieved marked local and distant responses after receiving a combination of low-dose RT, low-dose chemotherapy, mEHT, and low-dose immunotherapy. We present the following case in accordance with the CARE reporting checklist (available at https://dx.doi.org/10.21037/tro-21-16).

\section{Case presentation}

In 2012, a 51-year-old woman was diagnosed as having left-sided luminal A subtype BC (cT2N0M0), stage IIA. After receiving 2 cycles of neoadjuvant chemotherapy, the patient refused surgery and received only tamoxifen and naturopathic therapies for over 6 years. In July 2018, the patient finally agreed to undergo salvage left-sided modified radical mastectomy due to local progression by skin invasion and palpable axillary and supraclavicular nodes, she finally agreed for salvage left-sided modified radical mastectomy. Pathology reports revealed invasive ductal BC with chest wall and skin invasion, 11 positive lymphadenopathies, complicated by positive surgical margins, and extranodular extension, which indicated pT4bN3a, stage IIIB. The patient refused adjuvant treatments after surgery. In February 2019, she noticed multiple eruptions of left chest wall tumors that, through biopsy, were confirmed to be metastatic BC and phenotype transformation into triplenegative subtypes. She was salvaged through reoperation and cryotherapy.

In July 2020, the BC recurred with multiple left chest wall indurations and serous discharge as well as an enlarged axillary mass measuring more than $7 \mathrm{~cm}$. The patient was salvaged through RT administered at 50 Gy in 25 fractions to the left chest wall and regional nodes, in addition to a focal boost of 70 Gy administered in 35 fractions to the axillary mass. After 2 months, the patient sought a second medical opinion due to a rapid outburst of a right breast nodule measuring more than $5 \mathrm{~cm}$, as well as progressive exertional dyspnea. A positron emission tomography (PET) scan performed in October 2020 revealed a right breast tumor of more than $7 \mathrm{~cm}$ protruding from the skin along with numerous metastatic nodules over the right breast and left chest wall. The scan also revealed multiple metastatic lesions in the regional and distant lymph nodes, ribs, lungs, and adrenal glands; bilateral pleural effusions; and pericardial masses extending from the left to the right atrium. Cardiac echography revealed pericardial metastases and pericardial effusion (Figure 1). The patient refused intravenous chemotherapy but agreed to receive a combination of palliative RT, oral chemotherapy, mEHT, and low-dose immunotherapy.

Treatment began after pericardiocentesis with a low dose of ipilimumab $15 \mathrm{mg}(0.3 \mathrm{mg} / \mathrm{kg})$ and nivolumab $30 \mathrm{mg}(0.5 \mathrm{mg} / \mathrm{kg})$ given every 4 weeks, in addition to mEHT treatment in 1-hour sessions twice weekly. mEHT was applied using an EHY 2000+ device (OncoThermGmbH, Germany). The mEHT protocol involved a large treatment field implemented using a $30-\mathrm{cm}$ electrode covering the right breast and a small treatment field implemented using a 10 -cm electrode applied directly to the protruding mass on the right breast; the 2 electrodes were applied alternately. A radiofrequency of 13.56 MHz was used with an automatic, real-time tuning device to ensure a standard wave ratio was maintained. The intratumoral temperature was not measured because the temperature elevation inside a treatment field measured using a conventional thermocouple is typically $<2{ }^{\circ} \mathrm{C}(12)$. After 4 sessions of mEHT treatment, palliative RT at 20 Gy in 12 fractions given daily, was administered to the right ulcerative breast tumor, pericardial lesions, and rib metastatic lesions by tomotherapy (Accuray, Sunnyvale, CA, USA) (Figure 2). Chemotherapy was given concurrently by using low-dose capecitabine $500 \mathrm{mg}$ t.i.d. and vinorelbine 

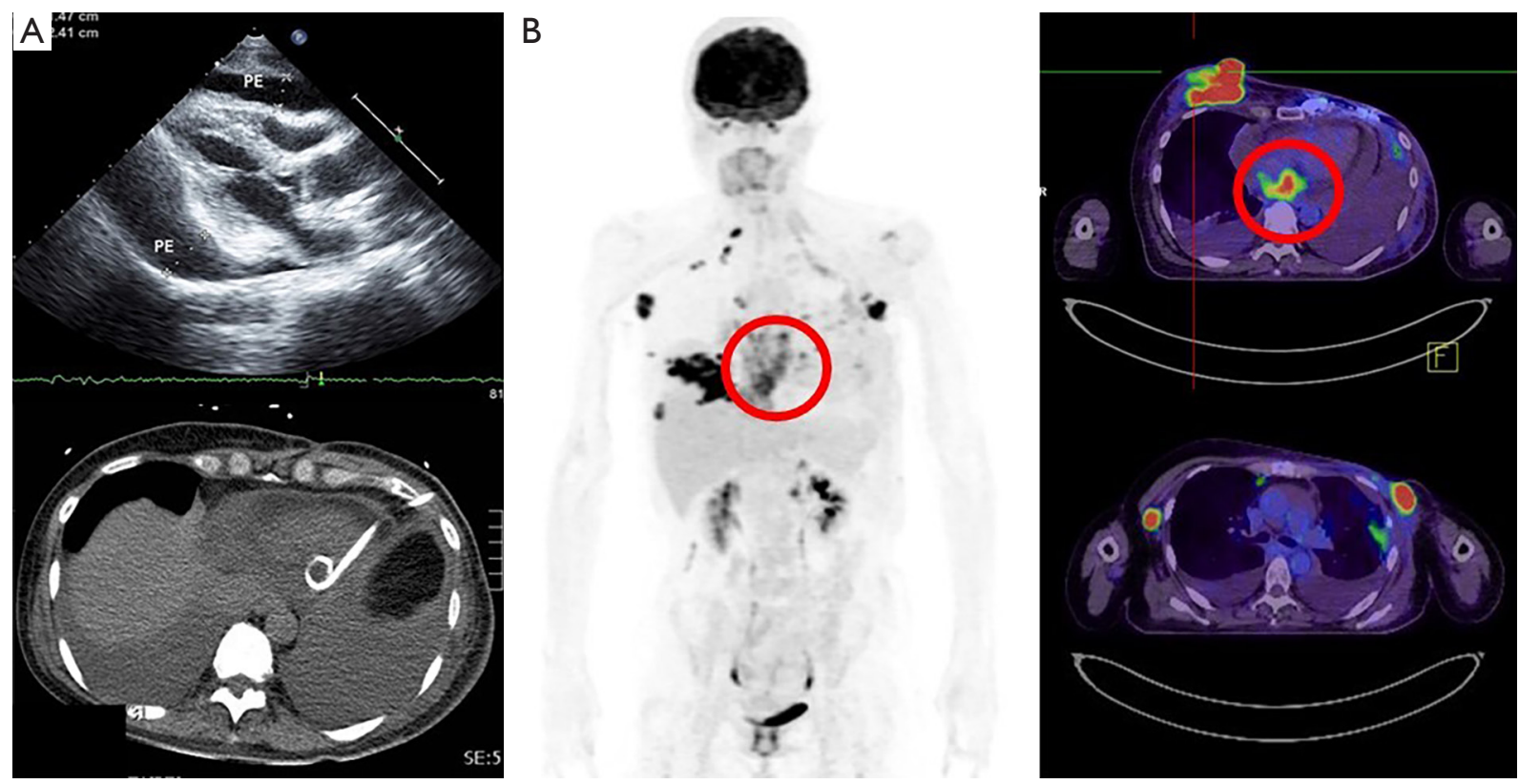

Figure 1 Heavily pretreated metastatic BC with (A) massive pericardial effusions treated through pericardiocentesis and drainage. (B) PET scan revealing multiple lesions involving the bilateral breast, pericardial (red circle), regional, and distant lymph nodes, ribs, lung, and adrenal glands with bilateral pleural and pericardial effusions. BC, breast cancer; PET, positron emission tomography.

$40 \mathrm{mg}$ weekly. The lesions of the left chest wall were not included in the RT field due to the short retreatment time and high in-field dose in previous treatment.

The patient refused oral chemotherapy 6 weeks after treatment was initiated. The protruding tumor of the right breast was nearly invisible at 3 weeks of treatment, and the patient was free from dyspnea after completing the RT course in December 2020. A PET scan 2 months after treatment revealed marked regression of the irradiated lesions and diminished pleural and pericardial effusions. Notably, the unirradiated left chest wall lesions and adrenal metastases outside the local treatment field demonstrated remote disease control (Figure 3). A posttreatment cardiac echocardiogram revealed diminished pericardium effusions without cardiac wall motion abnormalities. The patient continued the monthly low-dose immunotherapy protocol in conjunction with mEHT without any treatment-related toxicities. The patient's condition has remained stable for more than 6 months at the time of writing.

All procedures performed in studies involving human participants were in accordance with the ethical standards of the institutional and/or national research committee(s) and with the Helsinki Declaration (as revised in 2013).
Written informed consent was obtained from the patient for publication of this case report and accompanying images. A copy of the written consent is available for review by the editorial office of this journal.

\section{Discussion}

We present a case of $\mathrm{BC}$ with $\mathrm{CMs}$ that demonstrated an abscopal response more than 6 months after combination treatment involving low-dose immunotherapy, low-dose chemoradiotherapy, and HT. The prognosis of CMs is generally grave, with a median survival of 3.5 months if untreated $(4,5,13)$. Although patients with CMs typically present with disseminated disease, appropriate management can improve their quality of life and overall survival. Palliative RT is often underused due to the poor prognosis of CMs and the lack of randomized controlled trials $(5,6)$. In our case, we demonstrated that the combinatory treatment was effective and notable in that it produced a rapid and sustained abscopal response, demonstrated a radio- and immune-sensitization effect through HT, and involved of a low-dose immunotherapy protocol.

The patient was heavily pretreated; therefore, the 


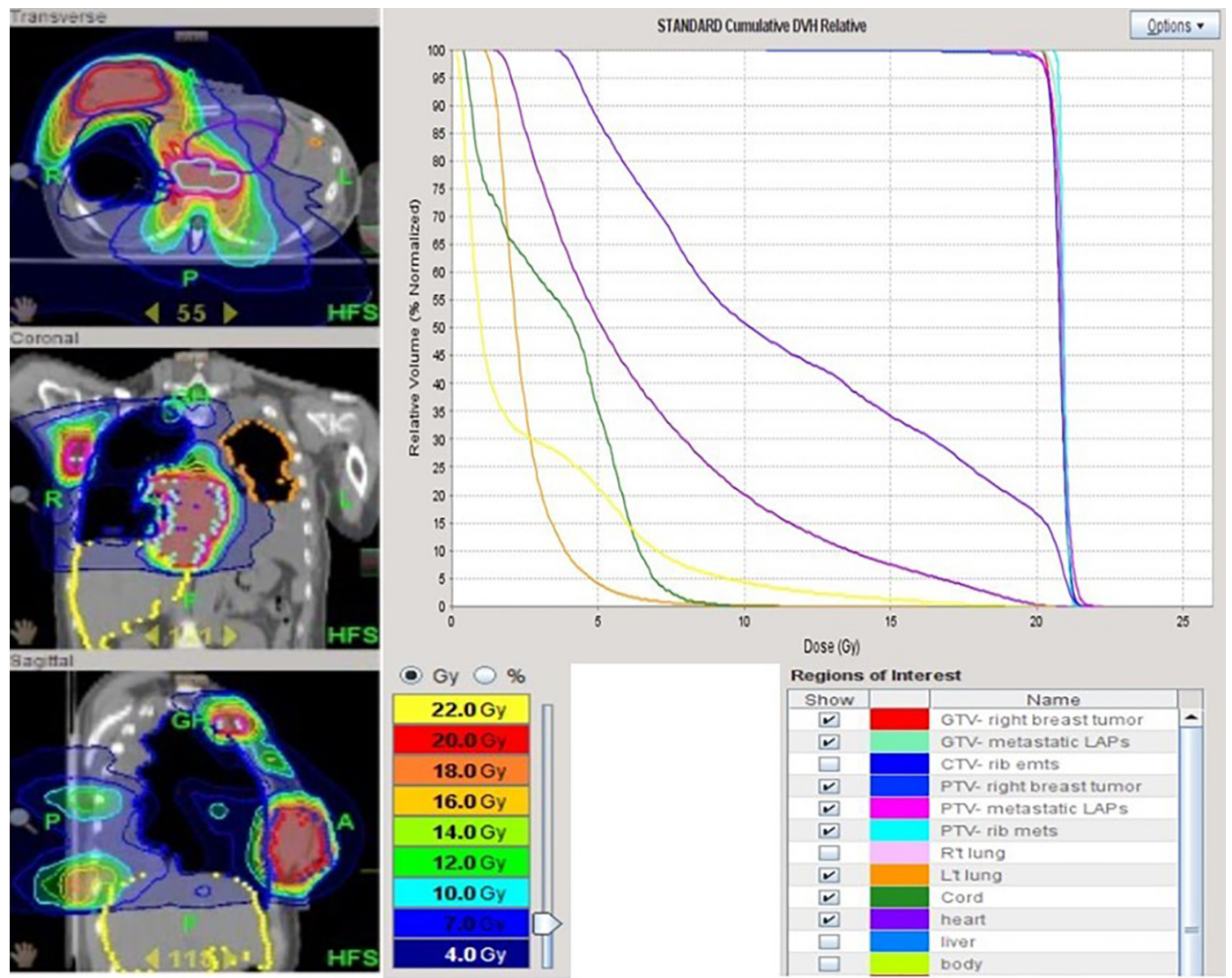

Figure 2 Dose-volume histogram, region of interest, and isodose curve. The RT plan was administered through tomotherapy at 20 Gy in 12 fractions to the right breast tumor, pericardial, and rib metastases. RT, radiotherapy; Gy, Gray.

response rate and duration of the response were expected to be low. Nevertheless, the observed local and distant responses to treatment were notably positive. Whether our case was a bona fide abscopal response is debatable because immunotherapy was applied. However, among the millions of patients treated with palliative RT between 1969 and 2014, only 46 cases were identified as abscopal events (14). Accordingly, we hypothesized that a rapid release of extracellular damage-associated molecular secretion patterns (DAMP) through RT and HT would facilitate tumor immunogenicity and enhance the efficacy of immunotherapy. Our patient exhibited a rapid response to treatment at the second week of RT, which supports a previous report that a rapid response may be correlated with abscopal events (11).

The prescribed RT dose was low when compared with conventional palliative RT courses administered at 30-35 Gy for 2-4 weeks. Despite the lower fractionated dose, the treatment response was robust and indicated a radiosensitization effect due to the combined HT. A study of metastatic melanoma treated with RT and immunotherapy revealed that, other than $\mathrm{HT}$, an RT fraction size of less than 3 Gy was the only factor associated with a high response rate (15). In our case, the protruding mass, especially the large-sized tumor, exhibited a stronger response than the smaller tumors did when treated with combined RT and mEHT (11). Larger tumors, usually situated near the surface of the body, absorb the radiofrequency energy more effectively. The absorbed energy results in prominent apoptosis, necrosis, and a release of DAMP, which may facilitate abscopal response $(7,11,16)$.

Immunotherapy along with chemotherapy was observed to prolong the overall survival of patients with metastatic triplenegative BC (7). For patients who refuse chemotherapy, a double checkpoint inhibitor blockade may be a sensible approach. Combination treatment involving an anti-CTLA4 

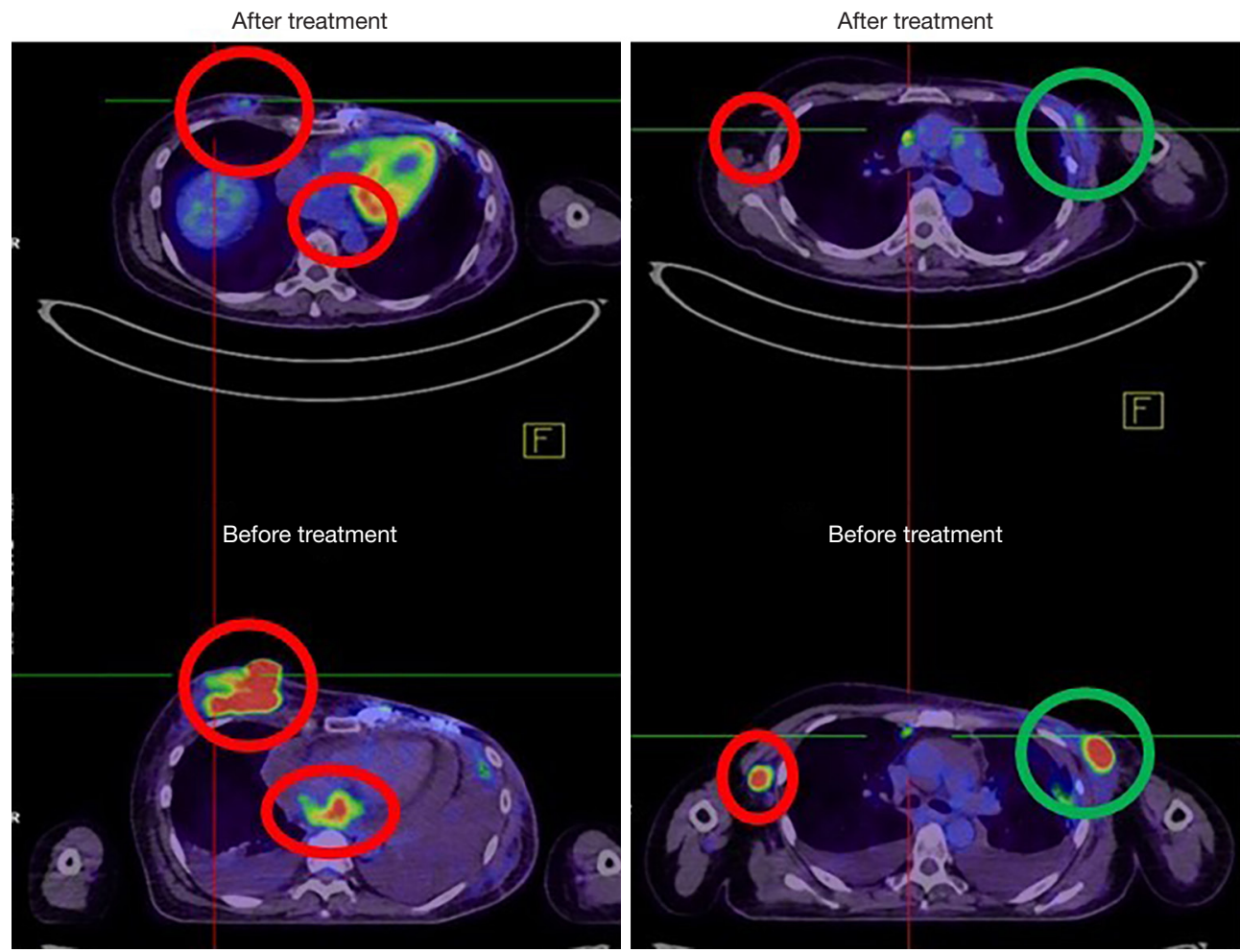

Figure 3 Red circle indicates the irradiated right breast mass and pericardial metastases with almost complete response. Green circle indicates the unirradiated left breast tumor with an abscopal response in the following image with diminished pericardial and pleural effusions.

antibody (ipilimumab) and anti-PD-1 antibody (nivolumab) was reported to achieve a 3 -year overall survival rate of $63 \%$ in metastatic melanoma but at the expense of a $58.5 \%$ rate of grade 3 or 4 immune-related adverse events (irAEs) (17). A meta-analysis of 80 clinical trials revealed that a combination of double checkpoint inhibitors (anti-CTLA-4 and antiPD-1 inhibitors) was associated with the highest rate of irAEs proportional to the prescribed dose (18). The concept of utilizing low-dose double immune checkpoint blockade was analyzed in a retrospective study of 131 metastatic patients who failed all conventional treatments (19). Treatment using low-dose ipilimumab $(0.3 \mathrm{mg} / \mathrm{kg})$ with nivolumab $(0.5 \mathrm{mg} / \mathrm{kg}), \mathrm{HT}$, and interleukin-2 was associated with an objective response rate of $31.3 \%$ and a 2 -year survival rate of $36.6 \%$. In addition, BC patients (including all phenotypes) demonstrated a $31 \%$ response rate, and the documented irAEs were mild (19).

The combination of low-dose chemoradiotherapy, lowdose immunotherapy, and HT is a safe option for palliative treatment. The observed rapid and sustained abscopal response renders this combination worthy of further study.
An official trial should be launched.

\section{Acknowledgments}

The authors thank the staff from the Department of Radiation Therapy and Oncology of Shin Kong Wu Ho-Su Hospital, especially Ms. Yi-Ying Huang and Ms. Yi-Chieh Li for their contributions through hyperthermia treatment. This manuscript was edited by Wallace Academic Editing. Funding: None.

\section{Footnote}

Provenance and Peer Review: This article was commissioned by the Guest Editors (Pei-Wei Shueng, Yen-Wen Wu and Long-Sheng Lu) for the series "Cardio-Oncology" published in Therapeutic Radiology and Oncology. The article has undergone external peer review.

Reporting Checklist: The authors have completed the CARE reporting checklist. Available at https://dx.doi. 
org/10.21037/tro-21-16

Conflicts of Interest: All authors have completed the ICMJE uniform disclosure form (available at https://dx.doi. org/10.21037/tro-21-16). The series "Cardio-Oncology" was commissioned by the editorial office without any funding or sponsorship. CJW serves as an unpaid editorial board member of Therapeutic Radiology and Oncology from May 2020 to Apr 2022. The authors have no other conflicts of interest to declare.

Ethical Statement: The authors are accountable for all aspects of the work in ensuring that questions related to the accuracy or integrity of any part of the work are appropriately investigated and resolved. All procedures performed in studies involving human participants were in accordance with the ethical standards of the institutional and/or national research committee(s) and with the Helsinki Declaration (as revised in 2013). Written informed consent was obtained from the patient for publication of this case report and accompanying images. A copy of the written consent is available for review by the editorial office of this journal.

Open Access Statement: This is an Open Access article distributed in accordance with the Creative Commons Attribution-NonCommercial-NoDerivs 4.0 International License (CC BY-NC-ND 4.0), which permits the noncommercial replication and distribution of the article with the strict proviso that no changes or edits are made and the original work is properly cited (including links to both the formal publication through the relevant DOI and the license). See: https://creativecommons.org/licenses/by-nc-nd/4.0/.

\section{References}

1. Bussani R, De-Giorgio F, Abbate A, et al. Cardiac metastases. J Clin Pathol 2007;60:27-34.

2. Al-Mamgani A, Baartman L, Baaijens M, et al. Cardiac metastases. Int J Clin Oncol 2008;13:369-72.

3. Catton C. The management of malignant cardiac tumors: clinical considerations. Semin Diagn Pathol 2008;25:69-75.

4. Cham WC, Freiman AH, Carstens PH, et al. Radiation therapy of cardiac and pericardial metastases. Radiology 1975;114:701-4.

5. Fotouhi Ghiam A, Dawson LA, Abuzeid W, et al. Role of palliative radiotherapy in the management of mural cardiac metastases: who, when and how to treat? A case series of 10 patients. Cancer Med 2016;5:989-96.

6. Arscott WT, Lal P, Mamtani R, et al. Long-term survival after treating cardiac metastasis with radiation and immune therapy: a case report. Cureus 2018;10:e2607.

7. Schmid P, Adams S, Rugo HS, et al. Atezolizumab and nab-paclitaxel in advanced triple-negative breast cancer. $\mathrm{N}$ Engl J Med 2018;379:2108-21.

8. Datta NR, Puric E, Klingbiel D, et al. Hyperthermia and radiation therapy in locoregional recurrent breast cancers: a systematic review and meta-analysis. Int J Radiat Oncol Biol Phys 2016;94:1073-87.

9. Lee SY, Fiorentini G, Szasz AM, et al. Quo Vadis oncological hyperthermia (2020)? Front Oncol 2020;10:1690.

10. Tsang YW, Huang CC, Yang KL, et al. Improving immunological tumor microenvironment using electrohyperthermia followed by dendritic cell immunotherapy. BMC Cancer 2015;15:708.

11. Chi MS, Mehta MP, Yang KL, et al. Putative abscopal effect in three patients treated by combined radiotherapy and modulated electrohyperthermia. Front Oncol 2020;10:254.

12. Lee SY, Kim JH, Han YH, et al. The effect of modulated electro-hyperthermia on temperature and blood flow in human cervical carcinoma. Int J Hyperthermia 2018;34:953-60.

13. Reynen K, Köckeritz U, Strasser RH. Metastases to the heart. Ann Oncol 2004;15:375-81.

14. Abuodeh Y, Venkat P, Kim S. Systematic review of case reports on the abscopal effect. Curr Probl Cancer 2016;40:25-37.

15. Chandra RA, Wilhite TJ, Balboni TA, et al. A systematic evaluation of abscopal responses following radiotherapy in patients with metastatic melanoma treated with ipilimumab. Oncoimmunology 2015;4:e1046028.

16. Storm FK, Harrison WH, Elliott RS, et al. Normal tissue and solid tumor effects of hyperthermia in animal models and clinical trials. Cancer Res 1979;39:2245-51.

17. Callahan MK, Kluger H, Postow MA, et al. Nivolumab plus ipilimumab in patients with advanced melanoma: updated survival, response, and safety data in a phase I dose-escalation study. J Clin Oncol 2018;36:391-8.

18. Shulgin B, Kosinsky Y, Omelchenko A, et al. Dose dependence of treatment-related adverse events for 
immune checkpoint inhibitor therapies: a model-based meta-analysis. Oncoimmunology 2020;9:1748982.

19. Kleef R, Nagy R, Baierl A, et al. Low-dose ipilimumab plus nivolumab combined with IL-2 and hyperthermia in

doi: $10.21037 /$ tro-21-16

Cite this article as: Chi MS, Wu JH, Shaw S, Wu CJ, Chen LK, Hsu HC, Chi KH. Marked local and distant response of heavily treated breast cancer with cardiac metastases treated by combined low dose radiotherapy, low dose immunotherapy and hyperthermia: a case report. Ther Radiol Oncol 2021;5:17. cancer patients with advanced disease: exploratory findings of a case series of 131 stage IV cancers - a retrospective study of a single institution. Cancer Immunol Immunother 2021;70:1393-403. 